\title{
Is There a Role for Direct Oral Anticoagulants in the Primary and Secondary Prevention of Myeloproliferative Neoplasm Associated Thrombosis?
}

\author{
Uzma Faruqi *D and Karen A. Breen * \\ Department of Haemostasis and Thrombosis, Guys and St Thomas' NHS Foundation Trust, London SE1 7EH, UK \\ * Correspondence: uzma.faruqi@gstt.nhs.uk (U.F.); karen.breen@gstt.nhs.uk (K.A.B.)
}

check for

updates

Citation: Faruqi, U.; Breen, K.A.

Is There a Role for Direct Oral

Anticoagulants in the Primary and

Secondary Prevention of

Myeloproliferative Neoplasm

Associated Thrombosis? Hemato 2021,

2, 769-780. https://doi.org/10.3390/

hemato2040053

Academic Editors: Martin Ellis and Claire Harrison

Received: 12 October 2021

Accepted: 12 December 2021

Published: 14 December 2021

Publisher's Note: MDPI stays neutral with regard to jurisdictional claims in published maps and institutional affiliations.

Copyright: (c) 2021 by the authors. Licensee MDPI, Basel, Switzerland. This article is an open access article distributed under the terms and conditions of the Creative Commons Attribution (CC BY) license (https:// creativecommons.org/licenses/by/ $4.0 /)$.

\begin{abstract}
Philadelphia chromosome negative myeloproliferative neoplasms (MPN) are clonal haematopoietic stem cell disorders. Of the MPNs, polycythaemia vera (PV) and essential thrombocythaemia (ET) confer a high thrombotic risk which may be the presenting feature of the disease. Thrombotic complications consist of both arterial and venous events and the presence of the JAK2 V617F mutation is associated with higher risk. Patients presenting with an unprovoked thrombus, particularly at an unusual site, e.g., splanchnic circulation, should be screened for the presence of this mutation. Historically, warfarin has been the only option for oral anticoagulation; however, there is now increasing evidence and practise to use direct oral anticoagulants (DOACs) in cancer. The seminal randomised control trials have demonstrated non-inferiority compared to low molecular weight heparin $(\mathrm{LMWH})$ with a preferable bleeding profile. DOACs are now the first line treatment for atrial fibrillation and venous thromboembolic disease, as recommended by NICE, and therefore there is increasing familiarity with these agents. Furthermore, there are now targeted antidotes available. This paper reviews evidence for efficacy and safety of DOACs in MPN. Whilst no randomised control trials have been performed, several retrospective studies and reviews of registry data have reproducibly demonstrated that, alongside cytoreduction, DOACs represent an effective modality of anticoagulation for treatment of venous thromboembolism in MPN. Furthermore, dosing regimens provide the option for longer term secondary prophylaxis. Use of DOACs in arterial thrombosis is an area for future development and there is already some evidence for utility in peripheral vascular disease.
\end{abstract}

Keywords: MPN; DOAC

\section{Introduction}

Myeloproliferative neoplasms (MPN) are clonal haematopoietic disorders characterised by myeloid progenitor proliferation in the bone marrow. Polycythemia vera (PV), essential thrombocythemia (ET) and primary myelofibrosis (PMF) are Philadelphia chromosome negative MPNs, as defined by the World Health Organisation (WHO) 2016 classification and diagnostic criteria [1]. Most patients will have a driver mutation, most commonly the JAK2 V617F mutation. In the absence of this mutation, CALR, MPL and JAK 2 exon 12 mutations will be present, with only a small proportion characterised as triple negative.

Thrombosis comprising both arterial and venous events are a common presenting feature and complication of PV and ET and there is an affiliation between the presence of the JAK2 V617F mutation and an increased thrombotic risk [2]. The presence of thrombosis automatically defines patients as high risk and increases both disease morbidity and mortality. Management comprises cytoreduction, anticoagulation as well as assessment and modification of any additional risk factors.

Molecular analysis of peripheral blood has allowed for rapid and accurate detection of driver mutations. This has transformed the diagnostic potential for MPNs, which is 
particularly relevant for patients presenting with normal blood counts and a seemingly unprovoked thrombotic event.

Management of cancer associated thrombosis has evolved over the last 5 years. Low molecular weight heparin $(\mathrm{LMWH})$ has historically been the most viable option; however, DOACs are now increasingly being used as first line anticoagulation. The seminal randomised control trials that have established the efficacy of DOACs in cancer are HOKUSAIVTE, SELECT-D, ADAM VTE and, most recently, the CARAVAGGIO study [3-6].

\section{Incidence}

The thrombotic risk is highest in PV (5.5 per 100 patient-years) followed by ET (1-3 per 100 patient-years) and PMF (2 per 100 patient years) [2,7]. At diagnosis, thrombosis is present in 20 to $50 \%$ of patients with PV and ET [8].

Arterial events, including myocardial infarction, peripheral arterial occlusions and strokes, comprise two-thirds of the thrombotic events observed in MPNs [2]. Of the onethird venous events, there is a preponderance for unusual site thrombosis, particularly cerebral sinus vein and splanchnic thrombosis as well as DVT and PE [9,10]. A total 50\% of Budd Chiari and 25\% of portal vein thromboses are associated with MPN [11]. JAK2 V617 screening is thus commonly performed in the assessment of unprovoked unusual site thrombosis and unexpected arterial thrombosis, i.e., where cardiovascular risk factors do not account for the clinical presentation.

Hulcratz et al. published a Swedish cohort study looking at thrombotic risk; 9429 MPN patients were compared to controls [12]. The results showed the risk of arterial and venous thromboses were significantly increased at all ages in MPN patients, with the highest risk at diagnosis and shortly afterwards. Arterial events were twice as common as venous events. This study corroborates the findings from previous studies looking at the incidence of thrombosis. The incidence is highest in PV, with approximately one third of patients initially presenting with a thrombotic event [2].

\section{Pathophysiology}

The development of thrombosis in MPN is multifactorial.

First published in 1978 in The Lancet, Pearson and Wetherley-Mein noted the correlation of thrombosis in PV with an elevated haematocrit [13]. The elevated haematocrit results in increased viscosity, and thus stasis, as well as increased marginalisation of platelets interacting with the vessel endothelium. This is corroborated by efficacy in achieving a target haematocrit $<0.45$ to minimise thrombotic risk.

The primary mechanisms are related to the prothrombotic tendency of the clonal haematopoietic progenitor cells and the proinflammatory state. Haematopoietic clonal expansion can result in chronic activation of the clotting cascade and a chronic inflammatory state resulting in vascular injury and impaired fibrinolysis creating a prothrombotic milieu. Hyperviscosity and increased proteases also contribute to the hypercoagulable state by compromising the integrity of the endothelium and resulting in increased expression of adhesion molecules.

\subsection{Leucocytosis}

Leucocytosis can be a common feature in MPN to varying degrees and it is difficult to ascertain the extent to which this is a contributing thrombotic risk factor. Landolfi et al. conducted a subanalysis of the ECLAP database and found that a white cell count $>15 \times 10^{9} / \mathrm{L}$ compared to those with a white cell count $<10 \times 10^{9} / \mathrm{L}$ was an independent thrombotic risk factor in PV, particularly for coronary artery disease [14]. Further assessment of this would be required to see if this risk is present in those with a well-controlled haematocrit and the contribution of additional cardiovascular risk factors. Observational studies have reported a greater correlation between arterial events and leucocytosis. 


\subsection{Thrombocytosis}

The role of thrombocytosis as a directly contributing factor is debated and has not been reproducible. The seminal ECLAP trial did not find a correlation between thrombosis and platelet count. Furthermore, extreme thrombocytosis has been associated with an acquired von Willebrand syndrome rather than a prothrombotic state. Whilst the absolute platelet number does not have a direct impact, platelet activation and aggregation are both factors to consider. The utility of aspirin in microvascular thrombosis in ET and managing cardiovascular risk in PV has directly reduced mortality.

\subsection{JAK2 V617F}

Several meta-analyses have reproducibly suggested that the JAK 2 V617F mutation is associated with a higher incidence of thrombosis, particularly in JAK2 V617 mutated ET. The aetiology, whilst not entirely understood, is likely to be multifactorial. A higher allelic ratio is considered to carry an increased risk of thrombosis and vascular complications. At a molecular level, platelets and neutrophils have been shown to express cell surface markers in keeping with increased activation, resulting in a hypercoagulable inflammatory state.

Immature or newly released platelets are considered to have increased haemostatic potential, with higher P selectin expression and an increased response to thrombin. Falanga and Marchetti report a correlation between the JAK2 V617F mutation and quantity of immature platelets in ET and PV patients [2]. This supports the role of myelosuppression in ameliorating the thrombotic potential.

\section{Primary Prophylaxis}

Primary prevention refers to the use of antithrombotic interventions in the absence of a confirmed thrombosis. Non-pharmacological methods, including optimisation of cardiovascular risk factors, smoking cessation and appropriate thromboprophylaxis during higher risk periods, e.g., peri-operatively, are critical in minimising the risk of thrombosis in this high-risk cohort.

For primary prophylaxis for arterial events, aspirin is an effective strategy, as demonstrated in the ECLAP study [14]. This trial looked at the efficacy of aspirin as primary prevention against thrombotic events in PV patients. There was a 60 percent decrease in the risk of the combined primary end point of myocardial infarction, stroke, major venous thrombosis or death from cardiovascular causes, which was statistically significant. It is thought that low dose aspirin suppresses the increased thromboxane synthesis in PV [15]. This is also seen in patients with acute coronary syndrome. The benefits and safety of aspirin in primary prevention against cardiovascular disease are well established outside of MPN [16].

Currently, there is no evidence to support a role for anticoagulation with DOACs in the absence of confirmed thrombosis as a primary prevention strategy. The exception to this would be in the presence of another significant additional risk factor, such as orthopaedic surgery resulting in reduced post-operative mobility, as this is the established standard of care regardless of comorbidities.

In patients with an inherited thrombophilia, there is no current evidence to support thromboprophylaxis in the absence of a confirmed thrombotic event.

The AIRPORT-MPN is a phase 2 study that is currently underway and due for completion in May 2022 [17]. The aim is to assess feasibility of thromboprophylaxis with apixaban $2.5 \mathrm{mg}$ twice daily in JAK2 positive MPN compared to aspirin. This will be beneficial to the cohort of patients intolerant to antiplatelet agents. It will be interesting to see whether this also improves the arterial and cardiovascular morbidity, as the studies looking at peripheral vascular disease have utilised a rivaroxaban $2.5 \mathrm{mg}$ twice daily regime.

\section{Vitamin K Antagonists}

Prior to DOACS, vitamin K antagonists (VKA) were the only oral anticoagulant option, with warfarin used most commonly. In those intolerant of warfarin, acenocoumarol is 
often utilised. The mechanism of action is via inhibition of the vitamin $\mathrm{K}$ epoxide reductase complex, thereby depleting the vitamin $\mathrm{K}$ that is available for the gamma carboxylation of vitamin $\mathrm{K}$ dependent cofactors, i.e., factors II, VII, IX, X, protein C and protein S.

Atrial fibrillation (AF) is the most common indication for anticoagulation and, prior to DOACS, all patients with AF were anticoagulated with warfarin. Much of the data on bleeding risk are therefore based on this cohort of patients. Current guidance is for DOACs to be utilised first line for VTE and AF and, for those on warfarin, conversion to a DOAC should be offered [18].

Patients on VKA have a 2-fold increase in bleeding rate [19]. The risk of major bleeding is up to $0.5 \%$ per year and intracerebral haemorrhage, which is the major cause of mortality and morbidities, is $0.2 \%$ per annum compared to controls [20]. Interestingly, non-traumatic DOAC related intracerebral haemorrhage is associated with a smaller volume bleed and a less severe neurological deficit as compared to VKA related intracerebral bleed [21].

From a pragmatic perspective, the impact of diet on warfarin metabolism can result in labile INRs. Furthermore, time in therapeutic range is a key measurement of treatment efficacy and frequent INR monitoring, if required, can be difficult and impact compliance. Home testing devices should be calibrated and all patients on VKA should continue under a warfarin clinic for monitoring. The time in therapeutic range can vary significantly, thus impairing the efficacy of anticoagulation for those with labile or persistently subtherapeutic INRs.

VKA are used most frequently for arterial thrombosis and can still be used for AF or VTE when a DOAC is contraindicated or it is patient preference. VKA are the only oral anticoagulant option licensed for metallic heart valves.

\section{DOACs in Cancer Thrombosis}

Commonly used direct oral anticoagulants comprise Xa inhibitors-rivaroxaban, edoxaban and apixaban —or the direct thrombin inhibitor dabigatran. All the Xa inhibitors have demonstrated non-inferiority compared to LMWH and warfarin, with a better bleeding profile in the management of VTE and cancer associated thrombosis (CAT) compared to warfarin. This is also the agent of choice for non-valvular atrial fibrillation with a CHADSVASC score of greater than or equal to two.

The benefits of DOACs include a fixed dosing schedule that does not require monitoring and a more predictable pharmacokinetic profile. There are fewer drug interactions and minimal impact on variations in diet, aside from rivaroxaban which should be taken with food. Factors to consider are sites of absorption, particularly with metastatic malignancy and primary metabolism, e.g., if primarily hepatic, such as with apixaban, this may not be preferable with hepatic metastases and or resection. There are online databases and mobile phone apps to, rapidly and easily, assess the interaction profile with systemic anti-cancer treatment and DOACs.

The HOKUSAI-VTE trial compared edoxaban with dalteparin in the treatment of symptomatic or incidental VTE. The results demonstrated that edoxaban is non-inferior to dalteparin, with a lower rate of recurrent VTE [3]. The rate of major bleeding was higher in the edoxaban group and gastrointestinal malignancy was a risk factor for this. A total of $15 \%$ of patients had a colorectal malignancy in each arm; however, upper gastrointestinal malignancies were underrepresented (6\% in the edoxaban arm and $4 \%$ in the dalteparin arm). A total of $20 \%$ of patients qualified for a dose reduction and $70 \%$ had treatment within 4 weeks

The SELECT-D study, published in 2018, showed the primary outcome of VTE recurrence demonstrated rivaroxaban had a lower rate of recurrent VTE as compared with LMWH [4]. There was a higher incidence of bleeding, particularly in oesophageal malignancy.

The ADAM VTE study compared dalteparin with apixaban in the treatment of CAT. A total of $70 \%$ were on concurrent systemic cancer treatment and more than half had metastatic disease [5]. Haematological malignancies were underrepresented. There was a reduced rate of recurrent VTE in the apixaban arm and major bleeding was the same. 
Patients with splanchnic circulation and cerebral sinus vein thrombosis were also included in this study, with no adverse outcomes on DOAC treatment, although numbers were small.

The CARAVAGGIO trial, published in 2020, assessed efficacy of apixaban compared to dalteparin for CAT and also demonstrated non-inferiority to dalteparin with no increased risk of major bleeding [6]. This was a larger study than ADAM VTE, with more than 500 patients in each arm. A total of $60 \%$ of patients were on treatment during the trial period, which is slightly lower than the other CAT studies; however, both arms were matched for this.

In all the CAT studies, the distribution was primarily solid tumour types-colorectal, pancreatic, lung and gynaecological. Upper gastrointestinal malignancies were underrepresented, usually accounting for 4-6\% of the total number of patients and haematological malignancies, and MPNs were also underrepresented. All studies have reproducibly demonstrated non-inferiority with some evidence for reduced incidence of recurrent VTE on DOACs which may relate to compliance.

In solid organ malignancy, anticoagulation is discontinued after 6 months of treatment in the event of remission or cure. Thrombosis is often a feature of advanced disease when life expectancy is likely to be limited and, therefore, 6 months treatment may exceed disease prognosis. After 6 months, secondary prevention can be utilised if treatment is ongoing or active disease is present. The chronicity of MPN suggests that, if the same principles are extrapolated to the management of MPN, long term anticoagulation will be the recommendation.

The studies for use of DOACs in CAT provide good evidence that this is a safe and effective option for VTE management in neoplasia. The mechanism of thrombosis in solid organ malignancies is separate to that observed in MPN; however, thrombotic potential is correlated with disease control in both cohorts of patients and there is no evidence to suggest the mechanism of action of DOACS would render them less effective in MPN for management of venous thromboembolism.

\section{Evidence for DOACs in MPN}

MPN-DOACS was a large retrospective study involving 19 centres, looking at the incidence of thrombosis and bleeding associated with the use of DOACs in MPN [22,23]. A total of 442 patients were included with a diagnosis of PV, ET and PMF who had received a DOAC for AF or VTE. The results showed 22 recurrent VTEs and 10 thrombotic events in patients anticoagulated for AF. There were 26 major bleeding events, the majority of which were gastrointestinal. PMF and the use of dabigatran were found to be significant risk factors. This observational study highlights that the risk of recurrent thrombotic events is higher in the MPN cohort as compared to the general population; however, the rate of recurrent thrombosis is similar to that reported on warfarin, suggesting non-inferiority.

The MPN-DOACS outcome of non-inferiority to warfarin is a key finding and provides a good foundation for use of DOACS in this cohort. The prothrombotic propensity in MPN is well established and, in uncontrolled disease, there remains a residual thrombotic risk regardless of the modality of anticoagulation.

Hamulyak et al. conducted a systemic review of VTE management in MPN7. Ten observational studies were included in the analysis, with a total of 1295 participants. A total of $88 \%$ were JAK2 V617F mutated. In terms of thrombotic history, $30 \%$ had a history of arterial thrombosis and $57 \%$ venous thrombosis; $8 \%$ had a history of both. A total of 623 patients were treated with antiplatelets, 467 with vitamin $\mathrm{K}$ antagonists (VKA) and 77 with DOACS. The addition of cytoreduction significantly reduced the risk of recurrent events compared to anticoagulation or antiplatelets alone. On review of recurrent events (arterial or venous), there were $17.6 \%$ in those on VKA and cytoreduction and $7.9 \%$ in those with DOAC and cytoreduction.

This systematic review contains a large number of patients and, whilst there were significantly more recurrent events on VKA compared to DOAC with cytoreduction, there were many more patients on VKA included in the study which may have skewed 
results. The key component is the reduced thrombotic risk with cytoreduction; therefore, anticoagulation is effective primarily in conjunction with disease modifying treatment.

Huenerbein et al. published their experiences of DOACs in 71 out of a cohort of $782 \mathrm{MPN}$ patients [24]. There were 9 arterial events and 62 VTE. A total of 45 patients (6 arterial, 39 VTE) were treated with a VKA and 26 ( 3 arterial, 23 VTE) with a DOAC. The majority of thromboses were venous in this cohort and the results supported the current data on the safety and efficacy profile of DOACS in MPN, with no significant difference detected in bleeding or recurrent events.

Curto-Garcia et al. published outcomes of 32 patients receiving a DOAC for an MPNassociated VTE, including $12 \mathrm{PV}, 9 \mathrm{ET}, 9 \mathrm{MF}$ and $2 \mathrm{MPN} / \mathrm{MDS}$ overlap [25]. A total of 25 were JAK V617F positive. There were 38 thrombotic events recorded, with 15 splanchnic thrombosis and 2 cerebral sinus vein thrombosis included in the analysis. A total of 6 patients had 2 thrombotic events. None of the patients had major bleeding across a median follow up period of 2.1 years. There were no recurrent VTE events, and one patient had evidence of mesenteric ischaemia.

Although this was a small study, the results support the use of DOACs, including in those with unusual site VTE. The absence of major bleeding is a key finding combined with no recurrent events on a DOAC. DOACs are the agent of choice for VTE outside of malignancy and increasingly so in this cohort as well. From an efficacy and pragmatic perspective, there is increasing evidence for DOACS in VTE.

Fedorov et al. published a retrospective cohort study comparing 30 patients with PV or ET [26]. A total of 19 were treated with DOACS and 11 with VKA. In each group, there was one patient with a recurrent event and there were no patients with major bleeding events in either group. Whilst this, too, is a small cohort study, this further supports non-inferiority of DOACS and, in particular, no recurrent events or major bleeding.

\section{Management of Venous Thrombosis}

The strategy of managing confirmed VTE is therapeutic anticoagulation and cytoreduction in the acute setting followed by thromboprophylaxis in the longer term.

Patients with an MPN and a subsequent VTE, or those diagnosed with MPN following a VTE, should be counselled to continue with long-term anticoagulation. After the initial 6 months of therapeutic anticoagulation, a secondary prophylaxis dose can be continued long-term thereafter if counts are well controlled. Apixaban $2.5 \mathrm{mg}$ twice daily and rivaroxaban $10 \mathrm{mg}$ daily are both licensed for secondary prevention.

The initial period of anticoagulation at a therapeutic dose can be reviewed and extended in the absence of well controlled disease. As with any intervention the risk and benefit profile should be assessed on an ongoing basis, particularly if there is a significantly increased bleeding risk later in life.

Factors to consider when determining the most appropriate anticoagulant options include drug interactions, splenomegaly, varices (particularly in the context of splanchnic circulation thromboses resulting in portal hypertension) and thrombocytopenia secondary to splenomegaly. Patients on cytoreductive therapy have frequent monitoring of full blood counts. Appropriate adjustment of anticoagulation dosing should be undertaken if there is progressive thrombocytopenia. Therapeutic anticoagulation with a platelet count $>50 \times 10^{9} / \mathrm{L}$ and prophylaxis with a platelet count $>30 \times 10^{9} / \mathrm{L}$ is acceptable unless there are other significant bleeding risk factors.

For patients with evidence of varices, a variceal banding programme is often undertaken prior to switching from LMWH to a DOAC.

Apixaban, rivaroxaban and edoxaban represent the Xa inhibitors and dabigatran is a direct thrombin inhibitor.

Dabigatran requires twice daily dosing and primarily renal metabolism [27]. Absorption is decreased with concomitant proton pump inhibitor or $\mathrm{H}_{2}$ antagonist. It can exacerbate gastro-oesophageal reflux symptoms. Apixaban or edoxaban would be preferable if reflux is present. Considerations with rivaroxaban include once daily dosing and 
the necessity of taking the $20 \mathrm{mg}$ daily dose with food to aid absorption. Apixaban requires a twice daily dosing regimen, both for treatment and ongoing secondary prevention, but can be used in end-stage renal disease. Caution should be exercised in the context of liver disease with deranged liver enzymes and hyperbilirubinaemia, particularly for apixaban and rivaroxaban due to hepatic metabolism.

\section{Management of Arterial Thrombosis}

The mainstay of managing arterial thrombosis is aggressive management of modifiable cardiovascular risk factors, particularly smoking, hypertension, hyperlipidaemia, diabetes and obesity.

The benefits of aspirin in the secondary prevention of cardiovascular disease are well established [28]. The seminal ECLAP study assessed the impact of low dose aspirin in PV [29]. The utility and efficacy of aspirin in MPN has been transformative. A total of 518 patients were randomised to low dose aspirin (100 $\mathrm{mg}$ daily) or placebo, and the primary end points were the incidence of non-fatal myocardial infarction, stroke, PE, major venous thrombosis or death from cardiovascular causes. The findings showed that age greater than 65 years and a previous thrombosis were the strongest predictors of a cardiovascular event. Other factors were smoking, hypertension and congestive cardiac failure. The use of aspirin was shown to reduce the risk of the thrombotic end points as compared to placebo.

DOACs are licensed as first line anticoagulants in venous thrombosis. There is now emerging data on the utility of DOACs in arterial disease.

The COMPASS study reported low dose rivaroxaban (2.5 $\mathrm{mg}$ twice daily) with aspirin in patients with stable atherosclerotic vascular disease showed improved cardiovascular outcomes [30]. There were more bleeding events as compared to those randomised to aspirin alone. This would be an area of future development and consideration in well controlled MPN associated arterial thrombosis with stable disease.

Similarly, VOYAGER-PAD, published earlier this year, looked at patients with peripheral artery disease undergoing lower limb revascularisation. The aim was to assess the efficacy of low dose rivaroxaban and looked at rivaroxaban $2.5 \mathrm{mg}$ BD with aspirin compared to aspirin alone. The primary end point was time to first event of a significant ischaemic event and the results demonstrated that the addition of rivaroxaban reduced events by $15 \%$.

Both VOYAGER-PAD and COMPASS provide evidence for peripheral vascular disease as a preventative strategy or in stable peripheral vascular disease. There is currently no data on the efficacy of DOACs in acute or embolic arterial thrombosis.

APPRAISE-2 was an RCT, assessing the addition of apixaban $5 \mathrm{mg}$ twice daily in addition to antiplatelet therapy in patients with a recent acute coronary syndrome and two other risk factors to assess if this would reduce the risk of recurrent ischaemic events. However, the study was terminated early due to an increase in major bleeding with apixaban without a significant reduction in recurrent ischaemic events.

Depending on the aetiology of the thrombosis, an antiplatelet alongside cytoreduction may be sufficient, e.g., for a coronary artery event. If, however, there is an isolated acute arterial thrombotic event, we would currently advocate the use of warfarin as the efficacy of DOACs in arterial disease in MPN is yet to be validated.

\section{Pregnancy}

Pregnancy and MPN both confer an increased thrombotic risk and therefore any additional modifiable risk factors should be aggressively managed.

Tefferi et al. report that $12 \%$ of the MPN Mayo Clinic case series of 3023 patients were women of childbearing potential and, of these, 50\% had a diagnosis of ET. PV and PMF are less common in women of childbearing age. In the UK, Robinson et al. report the incidence of MPN pregnancies is 3.2/100,000 maternities per year in the UK [31]. 
Thrombosis is a cause for maternal mortality in the normal population and the risk is increased in this cohort. All women should be offered aspirin and LMWH if there is any additional risk factor as per RCOG recommendations. All women should also be offered uterine artery doppler at 20/40 gestation. In high-risk pregnancies, interferon alpha can be considered and LMWH should be given throughout if there is a history of any prior thrombosis. All patients should continue LMWH post-partum.

Obstetric complications are similar to those seen in obstetric antiphospholipid syndrome, including miscarriages, late foetal loss, pre-eclampsia and intra-uterine growth restriction. There is also a lower live birth rate as compared to the general population, with $59 \%$ of foetal losses occurring in the first trimester.

Management of an acute thrombosis in pregnancy should be with therapeutic anticoagulation with LMWH in a twice daily pregnancy dosing schedule, alongside discussion with an MPN specialist regarding initiation of cytoreduction with pegylated interferon.

Patients on long-term anticoagulation should be counselled on the need to switch to LMWH at the point of a positive pregnancy test. We do not routinely advocate switching to LMWH prior to confirmation of pregnancy as the timescale can be extremely variable and there is no conclusive evidence of adverse outcome with DOAC exposure in the initial weeks [32]. Post-partum LMWH should be continued whilst breast feeding and DOACs can be resumed once no longer breast feeding due to the risk of transfer in breast milk.

Pregnancies should be managed in an experienced obstetric haematology unit. All patients should have an anaesthetic assessment and a clear plan, particularly regarding neuraxial analgesia in the context of anticoagulation.

\section{Bleeding Risk}

Bleeding risk in MPN is variable, with the most common source being gastro-intestinal [33]. This risk is increased in the context of portal hypertension, varices and/or thrombocytopenia related to disease or treatment. Unlike thrombosis, bleeding is less common at the point of diagnosis and tends to occur later in the trajectory of the disease [34].

Consideration should be taken for those on antiplatelets and anticoagulation simultaneously. Sometimes this is unavoidable, e.g., in the context of a cardiac stent; however, where thrombosis is chronic and MPN is well controlled, a secondary prophylactic DOAC dose can be used to minimise this. The availability of specific DOAC reversal agents should also ameliorate the impact of anticoagulation on major bleeding due to targeted and fast acting reversal-andexanet alpha for direct $X a$ inhibitors apixaban and rivaroxaban. Idaracizumab is available for dabigatran.

There are varying data on which DOACs are associated with an increased risk of bleeding. Guo et al. published a meta-analysis and reviewed 25 RCTs in total [35]. Their findings showed that the risk of major gastrointestinal bleeding was higher with rivaroxaban and dabigatran overall and apixaban and edoxaban had a more favourable bleeding profile in this context. Interestingly, Houghton et al. recently published a subanalysis of their gastrointestinal CAT cohort [36]. A total of 499 patients with a GI malignancy (208 lower GI luminal and 64 upper GI) were included as well as pancreatic and hepatobiliary, and they found the rate of major bleeding in GI cancer was similar to that in non-GI cancer for apixaban, rivaroxaban and enoxaparin. Interestingly, they found apixaban had a higher rate of major bleeding in patients with luminal GI cancer compared with enoxaparin. The increased hepatic metabolism of apixaban, particularly in the context of metastatic or obstructing disease, may also be a contributing factor to this.

Schaefer et al. looked at the impact on bleeding in 647 patients on a DOAC with aspirin [37]. Their study cohort had no clear indication for aspirin. Results showed that a combination of DOAC and aspirin resulted in a significantly higher rate of bleeding (319 bleeding events compared to 261 bleeding events). This was primarily non-major cutaneous, gastrointestinal or genitourinary bleeding events. For those requiring antiplatelets in combination with antithrombotics, the lowest risk of bleeding is with DOACs rather 
than warfarin and at a lower dose where possible (i.e., secondary preventative dose), in combination with aspirin [38].

Luminal and upper gastrointestinal malignancies have a higher bleeding risk for a number of reasons, including spontaneous bleeding from the tumour and ulceration. For MPN patients with varices or those on a banding programme, close monitoring is required during periods of therapeutic anticoagulation and parenteral heparin can be considered until the banding programme is complete.

Acquired von Willebrand syndrome should be considered in those with a very high platelet count, particularly if there are any symptoms of a bleeding diathesis.

Patients on long-term anticoagulation should be counselled regarding the need for a bridging plan prior to any planned interventions and to consult a haematologist prior to an emergency procedure if fully anticoagulated.

\section{Discussion}

Most of the current data are based upon retrospective studies. A number of these are in specialist centres. The key reproducible findings of no significantly increased risk of recurrent VTE events and no increased bleeding events support the efficacy of DOACs in this group. Indeed, major bleeding is reduced in cohorts treated with DOACs compared to warfarin. The data on use and efficacy of DOACs are supported by our experience in a large tertiary centre.

There is high quality evidence for DOACs in cancer and DOACs are the anticoagulant of choice where possible. The seminal DOAC studies did not incorporate any significant MPN incorporated; however, the principles of management can still be applied. From an evidence perspective, the gold standard would be a multi-centre randomised control trial comparing warfarin and DOACs in MPN. From a pragmatic perspective, DOACS have already been adopted in many centres internationally to treat MPN thrombosis with favourable results. Historically, there was more familiarity with warfarin as this was the only oral anticoagulant option for many years; however, as DOACs have become the first line for VTE and AF management, this is evolving.

Factors to take into account in anticoagulating MPN patients include the chronicity of the disease; median survival can be in excess of 30 years from diagnosis [39]. The mechanism of thrombosis is separate compared to other malignant diseases. The most beneficial strategy to ameliorate thrombotic potential has been proven to be cytoreduction alongside anticoagulation, improving both morbidity and mortality outcome. There is no current evidence to suggest DOACs would be rendered less effective based upon their mechanism of action in MPN. Whilst the mechanism of thrombosis in solid organ malignancy is separate to that in MPN, the principle of treating the underlying malignant process to minimise thrombotic risk can be extrapolated to the MPN cohort. Due to the chronic nature of MPN, patients with a confirmed VTE event should be counselled to continue long-term anticoagulation in the absence of any significant contraindications and this should be reviewed regularly.

DOACs have shown no increased bleeding risk and indeed provide the option of a secondary prophylactic dose reduction once disease stability has been established with cytoreduction and after completion of 6 months of full dose therapeutic anticoagulation.

As with any new agent, it is essential to consider drug interactions, side effect profile, pharmacokinetics and frequency of dosing. Data regarding bleeding risks with DOACs are conflicting and patients should be counselled on signs and symptoms of bleeding prior to initiating any anticoagulant.

Overall, for venous events, we would advocate the use of DOACs in the treatment and secondary prophylaxis of venous thromboembolism. Thus far, observational studies in MPN support this strategy and demonstrate non-inferiority to warfarin and LMWH. For arterial thrombosis, there is emerging evidence of the utility and efficacy of DOACs in stable peripheral vascular disease; however, currently, VKA are still utilised if antiplatelets 
alone are not sufficient. It would be interesting to assess the utility of DOACs in acute arterial events and stable arterial disease in the longer term

Future directions would include longitudinal studies to assess the efficacy of DOACs across long-term follow up in anticoagulated MPN patients. There is no current evidence to support use of DOACs in primary prophylaxis against VTE; this may be an area for future development.

Author Contributions: Conceptualization, K.A.B. and U.F.; writing—original draft preparation, U.F.; writing-review and editing, K.A.B.; supervision, K.A.B. All authors have read and agreed to the published version of the manuscript.

Funding: This research received no external funding.

Institutional Review Board Statement: Not applicable.

Informed Consent Statement: Not applicable.

Conflicts of Interest: The authors declare no conflict of interest.

\section{References}

1. Arber, D.A.; Orazi, A.; Hasserjian, R.; Thiele, J.; Borowitz, M.J.; Le Beau, M.M.; Bloomfield, C.D.; Cazzola, M.; Vardiman, J.W. The 2016 revision to the World Health Organization classification of myeloid neoplasms and acute leukemia. Blood 2016, 127, 2391-2405. [CrossRef]

2. Falanga, A.; Marchetti, M.; Schieppati, F. Prevention and Management of Thrombosis in BCR/ABL-Negative Myeloproliferative Neoplasms. Hamostaseologie 2021, 41, 48-57. [CrossRef]

3. Raskob, G.E.; van Es, N.; Segers, A.; Angchaisuksiri, P.; Oh, D.; Boda, Z.; Lyons, R.M.; Meijer, K.; Gudz, I.; Weitz, J.I.; et al. Edoxaban for venous thromboembolism in patients with cancer: Results from a non-inferiority subgroup analysis of the Hokusai-VTE randomised, double-blind, double-dummy trial. Lancet Haematol. 2016, 3, e379-e387. [CrossRef]

4. Young, A.M.; Marshall, A.; Thirlwall, J.; Chapman, O.; Lokare, A.; Hill, C.; Hale, D.; Dunn, J.A.; Lyman, G.H.; Hutchinson, C.; et al. Comparison of an Oral Factor Xa Inhibitor with Low Molecular Weight Heparin in Patients with Cancer with Venous Thromboembolism: Results of a Randomized Trial (SELECT-D). J. Clin. Oncol. 2018, 36, 2017-2023. [CrossRef]

5. McBane, R.D., 2nd; Wysokinski, W.E.; Le-Rademacher, J.G.; Zemla, T.; Ashrani, A.; Tafur, A.; Perepu, U.; Anderson, D.; Gundabolu, K.; Kuzma, C.; et al. Apixaban and dalteparin in active malignancy-associated venous thromboembolism: The ADAM VTE trial. J. Thromb. Haemost. 2020, 18, 411-421. [CrossRef] [PubMed]

6. Agnelli, G.; Becattini, C.; Bauersachs, R.; Brenner, B.; Campanini, M.; Cohen, A.; Connors, J.M.; Fontanella, A.; Gussoni, G.; Huisman, M.V.; et al. Apixaban versus Dalteparin for the Treatment of Acute Venous Thromboembolism in Patients with Cancer: The Caravaggio Study. Thromb. Haemost. 2018, 118, 1668-1678. [CrossRef] [PubMed]

7. Hamulyak, E.N.; Daams, J.G.; Leebeek, F.W.G.; Biemond, B.J.; Te Boekhorst, P.A.; Middeldorp, S.; Lauw, M.N. A systematic review of antithrombotic treatment of venous thromboembolism in patients with myeloproliferative neoplasms. Blood Adv. 2021, 5, 113-121. [CrossRef]

8. Pearson, T.C. The risk of thrombosis in essential thrombocythemia and polycythemia vera. Semin. Oncol. 2002, 29 (Suppl. S10), 16-21. [CrossRef]

9. Sant'Antonio, E.; Guglielmelli, P.; Pieri, L.; Primignani, M.; Randi, M.L.; Santarossa, C.; Rumi, E.; Cervantes, F.; Delaini, F.; Carobbio, A.; et al. Splanchnic vein thromboses associated with myeloproliferative neoplasms: An international, retrospective study on 518 cases. Am. J. Hematol. 2020, 95, 156-166. [CrossRef]

10. Rungjirajittranon, T.; Owattanapanich, W.; Ungprasert, P.; Siritanaratkul, N.; Ruchutrakool, T. A systematic review and metaanalysis of the prevalence of thrombosis and bleeding at diagnosis of Philadelphia-negative myeloproliferative neoplasms. BMC Cancer 2019, 19, 184. [CrossRef]

11. Falanga, A.; Marchetti, M. Thrombotic disease in the myeloproliferative neoplasms. Hematol. Am. Soc. Hematol. Educ. Program 2012, 2012, 571-581. [CrossRef]

12. Hultcrantz, M.; Bjorkholm, M.; Landgren, O.; Kristinsson, S.Y.; Andersson, T.M.L. Risk for Arterial and Venous Thrombosis in Patients with Myeloproliferative Neoplasms. Ann. Intern. Med. 2018, 169, 268. [CrossRef] [PubMed]

13. Pearson, T.C.; Wetherley-Mein, G. Vascular occlusive episodes and venous haematocrit in primary proliferative polycythaemia. Lancet 1978, 2, 1219-1222. [CrossRef]

14. Landolfi, R.; Marchioli, R. European Collaboration on Low-dose Aspirin in Polycythemia Vera (ECLAP): A randomized trial. Semin. Thromb. Hemost. 1997, 23, 473-478. [CrossRef] 
15. Landolfi, R.; Ciabattoni, G.; Patrignani, P.; Castellana, M.A.; Pogliani, E.; Bizzi, B.; Patrono, C. Increased thromboxane biosynthesis in patients with polycythemia vera: Evidence for aspirin-suppressible platelet activation in vivo. Blood 1992, 80, $1965-1971$. [CrossRef] [PubMed]

16. Ittaman, S.V.; VanWormer, J.J.; Rezkalla, S.H. The role of aspirin in the prevention of cardiovascular disease. Clin. Med. Res. 2014, 12, 147-154. [CrossRef] [PubMed]

17. Delluc, A. Assessing Feasibility of Thromboprophylaxis with Apixaban in JAK2-Positive Myeloproliferative Neoplasm Patients (AIRPORT-MPN). NCT04243122. Available online: https://clinicaltrials.gov/ct2/show/NCT04243122 (accessed on 5 December 2021).

18. Guidance, NICE (National Institate for Health and Care Excellence) Preventing Stroke in People with Atrial Fibrillation. Available online: http:/ / pathways.nice.org.uk/pathways/atrial-fibrillation2021 (accessed on 5 December 2021).

19. Shoeb, M.; Fang, M.C. Assessing bleeding risk in patients taking anticoagulants. J. Thromb. Thrombolysis 2013, 35, 312-319. [CrossRef]

20. Vinogradova, Y.; Coupland, C.; Hill, T.; Hippisley-Cox, J. Risks and benefits of direct oral anticoagulants versus warfarin in a real world setting: Cohort study in primary care. BMJ 2018, 362, k2505. [CrossRef] [PubMed]

21. Tsivgoulis, G.; Lioutas, V.A.; Varelas, P.; Katsanos, A.H.; Goyal, N.; Mikulik, R.; Barlinn, K.; Krogias, C.; Sharma, V.K.; Vadikolias, K.; et al. Direct oral anticoagulant- vs. vitamin K antagonist-related nontraumatic intracerebral hemorrhage. Neurology 2017, 89, 1142-1151. [CrossRef] [PubMed]

22. Barbui, T.; De Stefano, V.; Carobbio, A.; Iurlo, A.; Alvarez-Larran, A.; Vannucchi, A.M.; Palandri, F.; Harrison, C.; Sibai, H.; Griesshammer, M.; et al. Direct Oral Anticoagulants for Myeloproliferative Neoplasms (MPN-DOACs): Results from an International Study on 442 Patients. Blood 2020, 136 (Suppl. 1), 42-43. [CrossRef]

23. Barbui, T.; De Stefano, V.; Carobbio, A.; Iurlo, A.; Alvarez-Larran, A.; Cuevas, B.; Marín, F.F.; Vannucchi, A.M.; Palandri, F.; Harrison, C.; et al. Direct oral anticoagulants for myeloproliferative neoplasms: Results from an international study on 442 patients. Leukemia 2021, 35, 2989-2993. [CrossRef] [PubMed]

24. Huenerbein, K.; Sadjadian, P.; Becker, T.; Kolatzki, V.; Deventer, E.; Engelhardt, C.; Griesshammer, M.; Wille, K. Direct oral anticoagulants (DOAC) for prevention of recurrent arterial or venous thromboembolic events (ATE/VTE) in myeloproliferative neoplasms. Ann. Hematol. 2021, 100, 2015-2022. [CrossRef] [PubMed]

25. Curto-Garcia, N.; Doyle, A.J.; Breen, K.A.; McLornan, D.P.; Radia, D.H.; Hunt, B.J.; Ling, G.; Harrison, C.N. Outcomes of patients receiving direct oral anticoagulants for myeloproliferative neoplasm-associated venous thromboembolism in a large tertiary centre in the UK. Br. J. Haematol. 2020, 189, e79-e81. [CrossRef]

26. Fedorov, K.; Goel, S.; Kushnir, M.; Billett, H.H. Thrombosis in myeloproliferative neoplasms: Treatment outcomes of direct oral anticoagulants and vitamin K antagonists. Res. Pract. Thromb. Haemost. 2021, 5, e12574. [CrossRef]

27. Mega, J.L.; Simon, T. Pharmacology of antithrombotic drugs: An assessment of oral antiplatelet and anticoagulant treatments. Lancet 2015, 386, 281-291. [CrossRef]

28. Weisman, S.M.; Graham, D.Y. Evaluation of the benefits and risks of low-dose aspirin in the secondary prevention of cardiovascular and cerebrovascular events. Arch. Intern. Med. 2002, 162, 2197-2202. [CrossRef] [PubMed]

29. Landolfi, R.; Marchioli, R.; Kutti, J.; Gisslinger, H.; Tognoni, G.; Patrono, C.; Barbui, T. Efficacy and safety of low-dose aspirin in polycythemia vera. N. Engl. J. Med. 2004, 350, 114-124. [CrossRef]

30. Eikelboom, J.W.; Connolly, S.J.; Bosch, J.; Dagenais, G.R.; Hart, R.G.; Shestakovska, O.; Diaz, R.; Alings, M.; Lonn, E.M.; Anand, S.S.; et al. Rivaroxaban with or without Aspirin in Stable Cardiovascular Disease. N. Engl. J. Med. 2017, 377, 1319-1330. [CrossRef]

31. Robinson, S.E.; Harrison, C.N. How we manage Philadelphia-negative myeloproliferative neoplasms in pregnancy. Br. J. Haematol. 2020, 189, 625-634. [CrossRef]

32. Beyer-Westendorf, J.; Tittl, L.; Bistervels, I.; Middeldorp, S.; Schaefer, C.; Paulus, W.; Thomas, W.; Kemkes-Matthes, B.; Marten, S.; Bornhauser, M. Safety of direct oral anticoagulant exposure during pregnancy: A retrospective cohort study. Lancet Haematol. 2020, 7, e884-e891. [CrossRef]

33. Elliott, M.A.; Tefferi, A. Thrombosis and haemorrhage in polycythaemia vera and essential thrombocythaemia. Br. J. Haematol. 2005, 128, 275-290. [CrossRef]

34. Kaifie, A.; Kirschner, M.; Wolf, D.; Maintz, C.; Hänel, M.; Gattermann, N.; Gökkurt, E.; Platzbecker, U.; Hollburg, W.; Göthert, J.R.; et al. Bleeding, thrombosis, and anticoagulation in myeloproliferative neoplasms (MPN): Analysis from the German SAL-MPN-registry. J. Hematol. Oncol. 2016, 9, 18. [CrossRef] [PubMed]

35. Guo, W.Q.; Chen, X.H.; Tian, X.Y.; Li, L. Differences In Gastrointestinal Safety Profiles Among Novel Oral Anticoagulants: Evidence From A Network Meta-Analysis. Clin. Epidemiol. 2019, 11, 911-921. [CrossRef] [PubMed]

36. Houghton, D.E.; Vlazny, D.T.; Casanegra, A.I.; Brunton, N.; Froehling, D.A.; Meverden, R.A.; Hodge, D.O.; Peterson, L.G.; McBane, R.D.; Wysokinski, W.E. Bleeding in Patients with Gastrointestinal Cancer Compared with Nongastrointestinal Cancer Treated with Apixaban, Rivaroxaban, or Enoxaparin for Acute Venous Thromboembolism. Mayo Clin. Proc. 2021, 96, $2793-2805$. [CrossRef] 
37. Schaefer, J.K.; Li, Y.; Kong, X.; Alexandris-Souphis, T.; Ali, M.A.; Haymart, B.; Kaatz, S.; Kline-Rogers, E.; Kozlowski, J.H.; Krol, G.D.; et al. Impact of Adding Aspirin to Direct Oral Anticoagulant Therapy without an Apparent Indication. Blood 2019, 134, 787. [CrossRef]

38. Barnes, G.D. Combining antiplatelet and anticoagulant therapy in cardiovascular disease. Hematol. Am. Soc. Hematol. Educ. Program 2020, 2020, 642-648. [CrossRef]

39. Tefferi, A.; Barbui, T. Polycythemia vera and essential thrombocythemia: 2021 update on diagnosis, risk-stratification and management. Am. J. Hematol. 2020, 95, 1599-1613. [CrossRef] 